\title{
Multi-hop Efficent Protocol for ZigBee, Bluetooth Low- Energy and ANT Sensor nodes
}

\author{
Nuray At ${ }^{*}$, Hakan Şenel ${ }^{1}$, Raed S. M. Daraghma ${ }^{2}$ \\ ${ }_{1}^{1}$ Department of Electrical and Electronics Engineering Anadolu University, Turkey. \\ 2 Department of Electrical Engineering Palestine Technical University (P.T.U), Palestine. \\ * Corresponding author. Tel.: 00970599707545; email: raeddaraghma@yahoo.com \\ Manuscript submitted April 15, 2017; accepted July 5, 2017. \\ doi: $10.17706 /$ jcp.13.4.471-479
}

\begin{abstract}
In this work, we propose a multi-hop protocol for wireless personal area networks In particular, both distance and residual energy taken into consideration to select the primary node or forwarder. The cost function selects a parent node which has high residual energy and minimum distance to the sink. Residual energy parameters balance the energy consumption among the sensor nodes while distance parameter ensures successful packet delivery to the base station. We provide comparing energy consumption of Bluetooth Low Energy (BLE), ZigBee and ANT protocols in which a specific range and low power wireless sensor node periodically sends a data packet to a sink. Simulation results show that our proposed protocol maximizes the network lifetime and nodes stay alive for longer period, for the same transmitted power, radio frequency, packet size and data rate for the three protocols, ZigBee consumes less energy and offers longer network lifetime.
\end{abstract}

Key words: Personal are network, ZigBee, bluetooth low energy, residual energy, lifetime.

\section{Introduction}

Wireless Sensor Networks (WSNs) have been considered for many applications. Typically, a WSN consists of a number of sensing nodes that gather data from the surrounding environment and deliver it to a Base Station (BS). Each sensor node has limited computation capability and limited amount of energy. It is difficult or even impossible to replace a node's battery, especially in areas that cannot be linked easily. Therefore, reducing the network's energy consumption is one of the basic challenges in developing routing techniques for WSNs. Furthermore, fast energy exhaustions in some nodes might cause network divisions and shorten network lifetime. Hence, balancing energy consumption among the nodes is another fateful case to be considered. This paper deals with the above challenges by considering energy-efficient routing techniques. In WPANs, sensor nodes are operated with limited energy source. It is required to use minimum power for transmitting data from sensor nodes to BS. One of the major disadvantages of WPAN is to recharge the batteries. An energy efficient routing protocol is required to overcome this issue of recharging batteries. Many energy efficient routing protocols are proposed in WSN technology [1]-[3].

For the majority of the embedded electronics industry, the trend towards extremely low power technology is based on the use of batteries as the prime power source. Extremely low power technology, therefore, is synonymous with battery power and, in today's applications, this typically translates to single or dual cell supplies comprising of a coin cell battery such as the CC 2450 , XBase 2 and AP2 or a pair of alkaline AA/AAA batteries. For the popular CC 2450, XBase 2 and AP2, it is usable voltage rang drops to $2.5 \mathrm{~V}$ before the voltage 
begins to reduce significantly.

Many routing protocols can improve the performance of energy efficiency and network lifetime by introducing intelligent clustering methods for considering energy, etc. however, most of these energy efficient routing protocols used dynamic cluster heads. The communication range of sensor nodes is based on IEEE 802.15.4(WPAN) which is one of the transmission standards for WSNs. IEEE 802.15.4 typically extends up to $10 \mathrm{~m}$ in all directions [4].

In this paper, we present a multi-hop protocol to solve the communication range for sensor nodes; in this study we don't consider a cluster head, each sensor node contains information about the location of BS, distance between them, distance to the BS node, residual energy. Thus each node can make intelligent decision about the next hop (forwarder) based on the maximum residual energy and minimum distance under the practical sensor network.

The outline of the paper is as follows. In Section 2, the previous work on the subject is summarized. The proposed protocol is presented in Section 3 and its performance is evaluated through simulations via MATLAB in Section 4. Some concluding remarks are given in Section 5.

\section{Previous Work}

In [5] Multi-hop energy efficient protocol for heterogeneous wireless sensor network (MEEP) is a clustering protocol for two levels heterogeneous sensor network is proposed. The proposed protocol combines the idea of clustering and multi-hop communication. Heterogeneity is created in the network by using some nodes of high energy.

In [6] a multi-hop balanced clustering protocol is proposed. This protocol is based on K-means clustering and uses genetic algorithm for multi-hop communication among cluster heads. The methods which are used is protocol cause balanced and uniform energy dissipation in nodes and made them to have smooth death rate.[7] in this paper a multi-hop energy efficient routing protocol based on data controlling (MEEDC) is proposed. Work focuses on heterogeneity network and a sensitive data controlling. The rule of super nodes in the proposed heterogeneous network is connecting to $\mathrm{CH}$ and the BS. The control of data transmission tries to reduce the number of transmissions and thus considerable energy conservation is achieved. In [8] multi-hop hierarchical reactive routing algorithm named E2MHR (Energy Efficient Multi-hop Hierarchical Routing), which aims to achieve of energy efficiency through the use of both multi-hop routing and completely distributed based on random number.

In WPAN technology, numbers of routing schemes are proposed. In this section, we present some proposed routing protocols. In [9] Quwaider et al. presented a routing protocol which tolerates to changes the locations in the network. They used a store and forward mechanism to increase the likelihood of a data packet to reach successfully to sink node. Each sensor node has the capability to store a data packet. In source to destination route, each node stores data packet and transmits to next node (forwarder). Storing a data packet and then retransmitting causes more energy to consume and longer end to end delay. In [10] Nabi et al. proposed a protocol similar to store and forward mechanism. They integrate this store and forward scheme with Transmit Power Adaption (TPA). To control transmission power consumption, all nodes know their neighbors. Nodes transmit data with minimum power with a stable link quality. The most often used Medium Access Control (MAC) protocol used in WSNs is the standard IEEE 802.15.4 [11]. The MAC controls the access to shared communication medium in networks, which is the air in WSNs. The MAC guarantees a fair access distribution and collision free data transfer for all participants of the network. This a complex task with severe timing constraints. Implementations of the protocol trade off these requirements with functional or flexibility limitations. 


\section{System Model}

Many radio models are proposed in literature. We use first order radio model proposed in [11].Given in Fig. 1. Here, $L$ bit data packets are transmitted to a receiver $\left(R_{x}\right)$ located at a distance $d$ from the transmitter $\left(T_{x}\right)$. Eelec is the amount of energy needed in $T_{X}$ or $R_{x}$ hardware to send or receive data due to path loss and multipath fading phenomena that occur in wireless channels, $\mathrm{T}_{\mathrm{X}}$ is equipped with an amplifier. The amplifier has a gain of $\varepsilon \operatorname{Ld}^{n}$ where $n$ denotes the path loss exponent. Note that the value of the path loss exponent is between 2 and 4 in general.

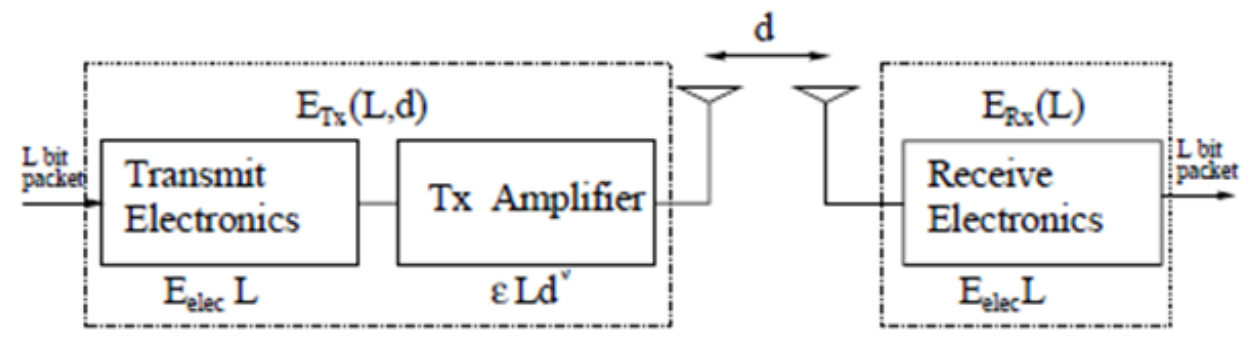

Fig. 1. Radio energy dissipation model [11].

$$
\begin{gathered}
E_{T x}(L, d)=E_{T x-\text { elec }}(L)+E_{T x-\text { amp }}(L, d) \\
E_{T x}(L, d)=E_{T x \text {-elec }} \times L+E_{T x-a m p} \times L \times d^{4} \\
E_{R x}(L)=E_{R x-\text { elec }} \times L
\end{gathered}
$$

where $E_{T x}$ is the energy consumed in transmission, $E_{R x}$ is the energy consumed by receiver, $E_{T x-e l e c}, E_{R x-e l e c}$ are the energies required to run the electronic circuit of transmitter and receiver, respectively. The energy parameters given in equations (1 and 2) depend on the hardware. We consider three transceivers used frequently in WPAN technology. The BLE node CC 2450 chip (Texas Instrument), AP2 transceiver modules (Nordic Semiconductor) were used for ANT, and the other transceiver is ZigBee. The operating frequencies of these transceivers are $2.4 \mathrm{GHz}$. The characteristics of these RF modules are given in Table 1.

Table 1. Characteristics of RF Modules

\begin{tabular}{|c|c|c|c|}
\hline Transceiver & ZigBee & BLE & ANT \\
\hline Rx sensitivity & $-102 \mathrm{~dB}$ & $-87 \mathrm{~dB}$ & $0 \mathrm{~dB}$ \\
\hline Tx Power & $0 \mathrm{dBm}$ & $0 \mathrm{dBm}$ & $2.4 \mathrm{GHz}$ \\
\hline Frequency & $2.4 \mathrm{GHz}$ & $2.4 \mathrm{GHz}$ & $\mathrm{AP2}$ \\
\hline Radio Chip & X BeeS2 & $\mathrm{CC} 2450$ & $250 \mathrm{~kb} / \mathrm{s}$ \\
\hline Bit rate & $250 \mathrm{~kb} / \mathrm{s}$ & $250 \mathrm{~kb} / \mathrm{s}$ & $100 \mathrm{byte}$ \\
\hline Packet size & $100 \mathrm{byte}$ & $100 \mathrm{byte}$ & 0.5 \\
\hline Number of nodes & 8 & 8 & $4 \mathrm{~nJ} / \mathrm{bit}$ \\
\hline Initial & 0.5 & $4 \mathrm{~nJ} / \mathrm{bit}$ & $2.5 \mathrm{pJ} / \mathrm{b} / \mathrm{m}^{4}$ \\
\hline$E_{T X-a m p}$ energy of node $\left(\mathrm{E}_{0}\right)$ & $4 \mathrm{~nJ} / \mathrm{bit}$ & $1.57 \mathrm{pJ} / \mathrm{b} / \mathrm{m}^{4}$ & $\begin{array}{c}\text { Two ray ground } \\
\text { (multi-path) }\end{array}$ \\
\hline$\varepsilon_{m p}$ & $0.049 \mathrm{pJ} / \mathrm{b} / \mathrm{m}^{4}$ & $\begin{array}{c}\text { Two ray ground } \\
\text { (multi-path) }\end{array}$ & Homogenous \\
\hline Propagation model & $\begin{array}{c}\text { Two ray ground } \\
\text { (multi-path) }\end{array}$ & Homogenous & $3.3 \mathrm{~V}$ \\
\hline
\end{tabular}

The power attenuation is depending on the distance between the transmitter and receiver; the 
propagation loss can be modeled as inversely proportional to $\mathrm{d}^{2}$ (free space model for short distances) or to $\mathrm{d}^{4}$ (multi path model for longer distances). In this study we used the multipath fading model $\left(\mathrm{d}^{4}\right)$.

From the parameters given in Table 1 we can find the radio energy parameters, the transmit energy per bit is given by:

$$
\begin{gathered}
E_{T x-a m p}=\frac{P_{t}}{R_{b}} \\
\varepsilon_{m p}=\frac{P_{r}}{R_{b} G_{t} G_{r} h_{t}^{2} h_{r}^{2}}
\end{gathered}
$$

where $\varepsilon_{m p}$ is the energy require for the amplifier circuit, this parameter will depend on the required receiver sensitivity and the receiver noise figure, as the transmit power needs to be adjusted that the power at the receiver is above a certain threshold. Where $P_{r}$ is the sensitivity of the receiver, $G_{t}$ is the gain of a transmitting antenna, $G_{r}$ is the gain of a receiving antenna, $\lambda$ is the wavelength of the carrier signal, $h_{t}$ is the height of the transmitted antenna above the ground and $h_{r}$ is the height of the received antenna above the ground. In the simulations following values are given ( $\left.G_{t}=G_{r}=1, h_{t}=h_{r}=1.5 \mathrm{~m}, R_{b}=250 \mathrm{Kbps}\right)$ into equations 3 and 4 given in Table 1.

\subsection{Proposed Protocol}

In this section, we present a new routing protocol for WPANs. The limited numbers of nodes in WPANs give opportunity to relax constraints in routing protocols. We improve the network stability period and throughput of the network. Next subsections give details of the system model and proposed protocol. In this scheme, we deploy eight sensor nodes on region. All sensor nodes are consumed to have equal energy and similar computation capabilities. BS is placed at the center of the square region. Fig. 2 shows the placement of eight nodes and BS on the square region $(50 \times 50 \mathrm{~m})$.

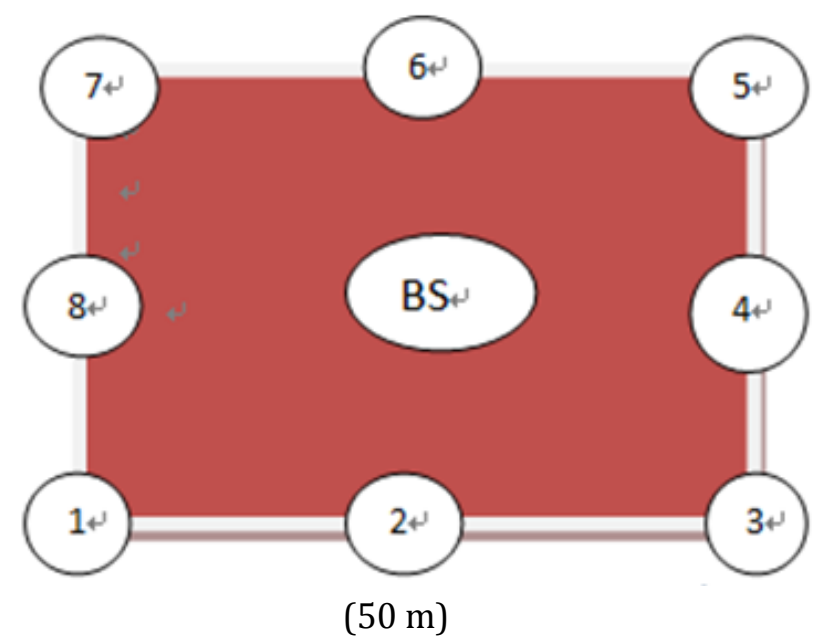

Fig. 2. An illustration of the network.

In order to save energy and to enhance network throughput, we proposed a multi-hop scheme for WPAN. In the following, we present a selection criterion for a node to become primary node or forwarder. To balance energy consumption among sensor nodes and to reduce energy consumption of the network, our proposed algorithm selects new forwarder in each round. BS node knows the ID, distance and residual energy status of 
the nodes. BS computes a cost function for all nodes and in the network let needs know these costs. On the basis of this cost function, each node decides whether to become forwarder node or not. The cost function of node $i$ is compute as follows:

$$
C(i)=\frac{d(i)}{\operatorname{Re}(i)}
$$

where $d(i)$ is the distance between the node $i$ and the BS, $\operatorname{Re}(i)$ is the residual energy of node $i$. A node with minimum cost function is preferred as a forwarder. All the neighbor nodes stick together with forwarder node and transmit their data to forwarder node aggregate data and forward to the BS. Forwarder node has maximum residual energy and minimum distance to BS; therefore, it consumes minimum energy to forward data to the BS.

\subsection{Path Loss Model}

Path loss represents the signal attenuation and is measured in decibel (dB). Signal power is also degraded by Additive White Gaussian Noise (AWGN). Path loss is the difference between the transmitted power and received power. Path loss occurs due to the increasing surface area of propagating wave front. Transmitting antenna radiates power outward and any object between transmitter and receiver causes destruction of radiated signal. Path loss is related to the distance and frequency and expressed as:

$$
P L(f, d)=P L(f) \times P L(d)
$$

The propagation model including both path loss model and long-normal shadowing is used in our simulations. In this model, the received power at distanced $\left(P_{r}(d)\right)$ is represented by:

$$
\left[P_{r}(d) / P_{r}\left(d_{0}\right)\right]_{d B}=-10 n \log _{10}\left(\frac{d}{d_{0}}\right)+X_{d B}
$$

where $P_{r}\left(d_{0}\right)$ is the received power at a reference distance and it is expressed as:

$$
P_{r}\left(d_{0}\right)=10 \log _{10} \frac{(4 \pi \times d \times f)^{2}}{c}
$$

where $f$ is the operating frequency, $c$ speed of light, $d$ is the distance between the transmitter and receiver. The value of the reference distance $d_{0}$ is $0.1 \mathrm{~m}, n$ is the path loss exponent, and $X_{d B}$ is a zero-mean Gaussian random variable representing the shadowing. In our simulations, the path loss exponent is set to 4 and the shadowing deviation is set to 7.4, this corresponds to a harsh environment with many obstacles [12].

\section{Simulation Results}

To evaluate the proposed protocol, we have conducted an extensive set of experiments using MATLAB with three transceiver modules. The performance evaluation of the proposed network is done with respect to the following parameters:

Stability period: The time interval between the start of the network operation and the death of the first sensor node also known as stable region.

Network lifetime: The time interval between the start of the network operation and the death of the last sensor node.

Number of dead nodes: The number of sensor nodes that have consumed all of their energy and are not 
able to do any kind of functionality.

Throughput: The rate of data sent from sensor nodes to the base station.

\subsection{Network Life Time}

Table 2 shows the average network lifetime of the proposed scheme. The proposed new cost function to select a forwarder node plays an important role to balance the energy consumption among the sensor nodes. New forwarder in each round is selected based on computed cost function. Table 2 clearly depicts the average number of dead nodes per round indicating stability time of the networks. The death of the first node occurs at the round 2962 in ZigBee whereas the death of the first node occurs at rounds 94 and 59 in BLE and ANT, respectively. The death of the last node occurs at the round 7676in ZigBee protocol whereas the death of the last node occurs at rounds 255and 160 in BLE and ANT, respectively. Hence, ZigBee protocol has better stability time and network lifetime as compared to the other protocols.

Table 2. The Round When First and Last Node Dies

\begin{tabular}{|c|c|c|c|}
\hline Protocol & ZigBee & BLE & ANT \\
\hline First dead node(round) & 2962 & 94 & 59 \\
\hline All dead node(round) & 7696 & 255 & 160 \\
\hline
\end{tabular}

Throughput The throughput is defined to be the successful packet received at the BS. WPAN has data to send, requires a protocol which has minumum packet drop and maximum successful data received by BS. ZigBee protocol achieves high throughput than BLE and ANT protocols, as shown in Fig. 3. The average number send to BS depends on the number of alive nodes. More alive nodes send more packets to BS which increases the throughput of network. The stability period for both ANT and BLE protocols are shorter than the ZigBee protocol implying packets sent to BS decreased. Hence, throughput of ANT and BLE is less. On the other hand, ZigBee protocol achieves high throughput due to longer stability period.

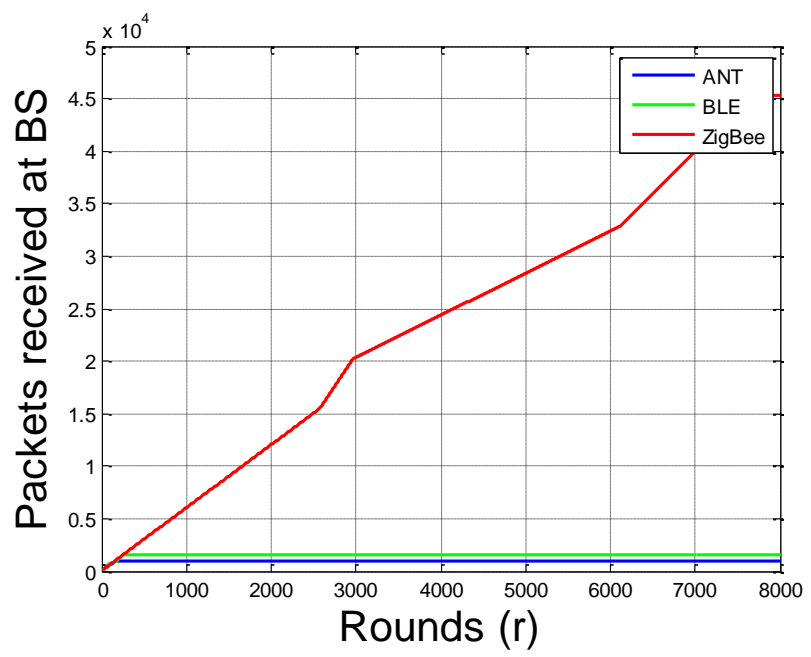

Fig. 3. Throughput.

Residual Energy The average energy of the network consumed at each round is presented in Fig. 4. The proposed scheme uses multi-hop topology, in which farthest node transmits its data to BS through a forwarder node. Forwarder node is selected using a cost function. Selection of appropriate forwarder in each round contributes to save energy. To transfer packets to BS, our multi-hop topology use different forwarder 
node in each round. Simulation results show that ZigBee protocol consumes minimum energy among BLE and ANT protocols. It means, in stability period, more nodes have enough energy and they transmit more data packet to BS.

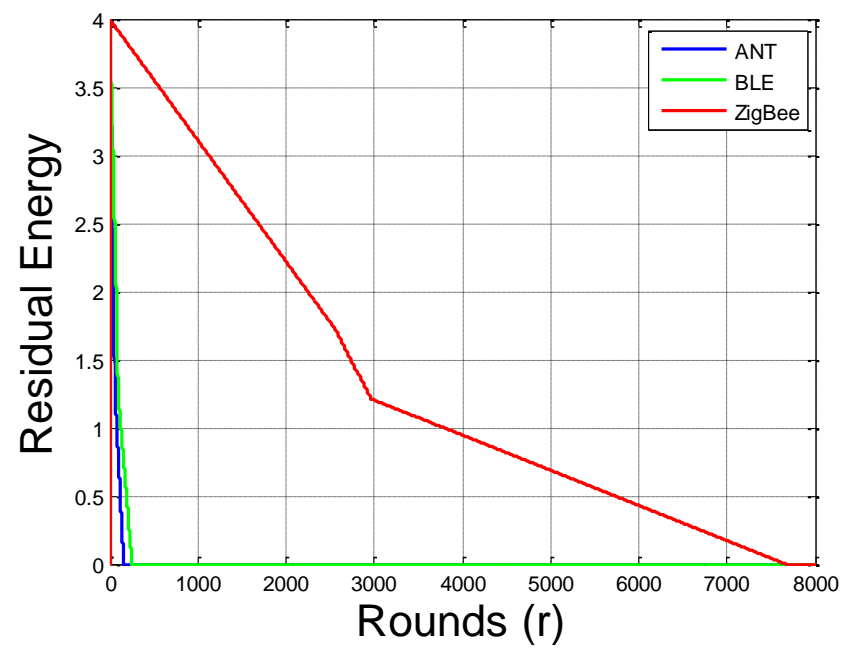

Fig. 4. Energy consumption.

\subsection{Path Loss}

Fig. 5 presents the path loss of different sensors. Path loss is afunction of frequency and distance. Path loss shown in Fig. 5 is a function of distance. It is calculated from its distance to BS with constant frequency 2.4 GHz. We use path loss coefficient 4 and 7.4 for standard deviation.

Path loss for BLE and ANT protocols are almost the same, but the ZigBee protocol has more path loss than the others due to the fact that the nodes in ZigBee need more times to die.

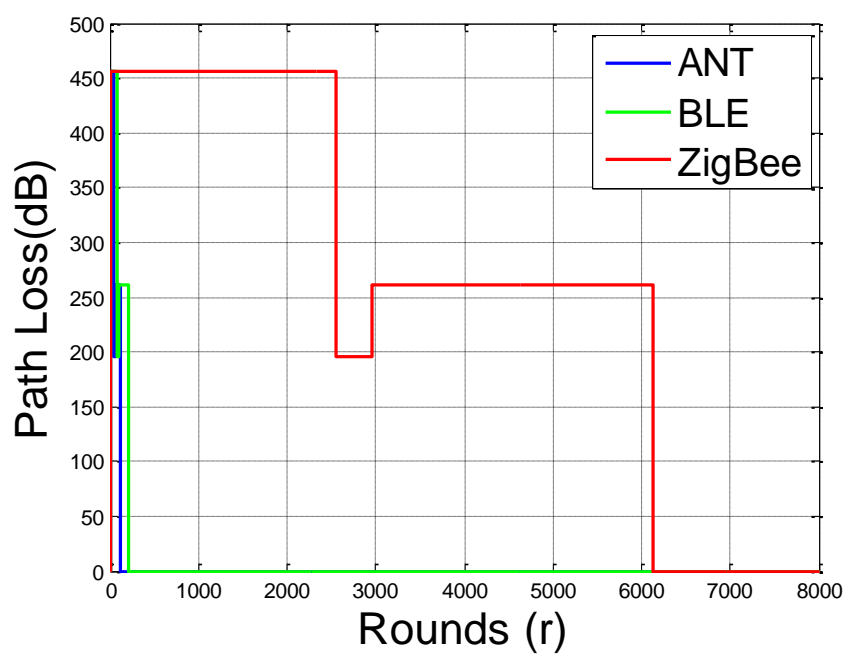

Fig. 5. Network path loss.

\section{Conclusion}

In this paper, we propose a new algorithm to route data in WPANs. The proposed scheme uses a cost function to select appropriate route to BS. Cost function is calculated based on the residual energy of nodes and their distance from BS. In this paper we analyzed the lifetime, throughput, energy consumption and the 
path loss for the ANT, ZigBee, and BLE protocols. We found that ZigBee protocol achieved the longest lifetime, throughput and lowest energy consumption, followed by BLE and ANT.

The results of this study should not be generalized to other scenarios. Furthermore, the lifetime, throughput, energy consumption and path loss of theses protocols might changes depending on other factors such as packet size variations, transmitter and receiver distances. In the future work we want to extend our work for larger WSN, we want use the cost function to select the optimal number of cluster head on the basis of residual node energy and the distance.

\section{References}

[1] Javaid, N., Qasim, U., Khan, Z. A., Khan, M. A., Latif, K., \& Javaid, A. (2013). On energy efficiency and delay minimization in reactive protocols in wireless multi-hop network. Proceedings of the 2nd International Conference on Saudi International Electronics, Communications and Photonics (SIECPC 13).

[2] Manzoor, B., Javaid, N., Rehman, O., Akbar, M., Nadeem, Q., Iqbal, A., \& Ishfaq, M. (2013). Q-LEACH: A new routing protocol for WSNs. Proceedings of International Workshop on Body Area Sensor Networks (BASNet-2013) in conjunction with the 4th International Conference on AmbientSystems, Networks and Technologies (ANT 2013), Halifax, Nova Scotia, Canada, Procedia Computer Science (pp. 926-931).

[3] Javaid, N., Khan, R. D., Ilahi, M., Ali, L., Khan Z. A., \& Qasim U. (2013). Wireless proactive routing protocolsunder mobility and scalability constraints. J. Basic. Appl. Sci. Res., 3(1), 1187-12001.

[4] Gutierrz, J. A., Naeve, M., \& Callaway, E. (2001). A developing standard for low-power low-cost wireless personal area networks. IEEE Network, 15, 12-19.

[5] Kumar, S., Prateek, M., \& Ahuja, N. J. (2014). MEEP: Multihop energy efficient protoccol for heterogenous wireless sensor network. International Journal of Computer Science and Telecomnication, 5, 3.

[6] Shiva, R., Manijeh, K., \& Reza, J. (2014). MBC: A multi-hop balanced clustering routing protocol for wireless sensor networks. International Journal of Artificial Intelligence and Mechatronics, 2(6), 264-270.

[7] Slahedine, C., Majed, A., \& Rafik, B. (2013). Multi-hop energy efficient routing protocol based on data controlling for wireless sensor network. IEEE Wireless Sensor.

[8] Chandrasekran, V., \& Shanmugam, A. (2013). An energy efficient multi-hop hierarchical routing in wireless sensor networks. JOSR Journal of Electronics and Communication Engineering, 5(4), 61-65.

[9] Quwaider, M., \& Subir, B. (2009). On-body packet routing algorithms for body sensor networks. Proceedings of the 1st International Conference on Networks and Communications.

[10] Nabi, M., et al. (2010). A robust protocol stack for multi-hop wireless body area networks with transmit power adaptation. Proceedings of the 5th International Conference on Body Area Networks (pp. 1-7).

[11] Heinzelman, W. R., Chandrakasan, A., \& Balakrishnan, H. (2000). Energy-efficient communication protocol for wireless microsensor networks. Proceedings of the 33rd Annual Hawaii International Conference on System Sciences.

[12] Ibrahim, R., Ho, Q. D., \& Tho, L. N. (2013). An energy-efficient and load cluster-based routing algorithm for CSMA-based wireless sensor networks. Proceedings of IEEE 77th Vehicular Technology Conference.

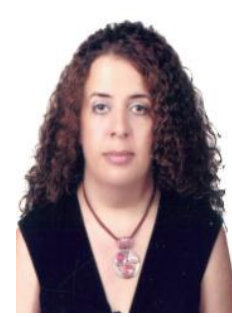

Nurray AT was born in 1972 in Turkey, she received her master degrees in electrical engineering from University of California, Davis 1998, she graduated her Ph.D from Anadolu University in 2005. She has published a number of papers in some journals and conferences and got around applause, she is one of the academic leaders of electrical engineering and many postgraduate are being in her door. 
Her mainly research areas include wireless channels with multiple antennas, signal processing and information security.

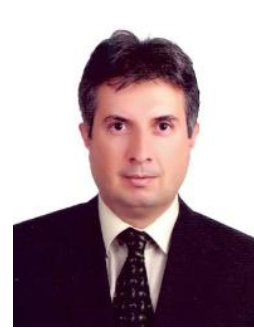

HAKAN Güray ŞENEL was born in 1968 in turkey, he received his master and Ph.D degrees in electrical and computer engineering from Vanderbilt University, USA (1997), he has published a number of papers and journals and conferences. He is one of the academic leaders of electrical engineering and many postgraduates are being in his door. His mainly research areas include image processing, wireless, optical communications and data communications.

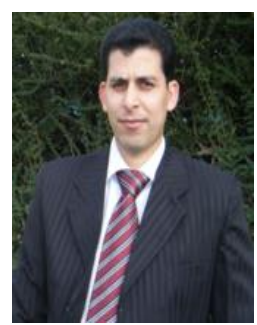

RAED S. M. DARAGHMA was born in 1977 in Palestine, he received his master degree from Jordan science and technology in electrical and communication engineering Jordan in 2010, he got his Ph.D degree from Anadolu University, Turkey in 2016. He has published a number of papers and journals. He was engaged in educational work many years, and mainly teaches digital communication, mobile, digital communication networks etc. at Palestine Technical University P.T.U. His mainly research areas include wireless sensor network, signal processing and electromagnetic waves. 